\title{
Protection of Factor Xa from Neutralization by the Heparin-Antithrombin Complex
}

\author{
Jerome M. Teitel and Robert D. Rosenberg, Dana-Farber Cancer Institute and \\ Beth Israel Hospital, The Harvard Medical School, Department of Biology \\ and Whitaker College, Massachusetts Institute of Technology, \\ Boston, Massachusetts 02215
}

\begin{abstract}
A BSTRACT We have studied the accessibility of Factor Xa to neutralization by the heparin-antithrombin complex within plasma and whole blood. This serine protease was detected by measuring the concentrations of activation fragments $\left(F_{2} / F_{1+2}\right)$ cleaved from prothrombin. The levels of $\mathrm{F}_{2} / \mathrm{F}_{1+2}$ were quantitated by means of a sensitive and specific radioimmunoassay. Our findings indicate that the binding of Factor $\mathrm{Xa}$ to "activated" platelets but not to phospholipid micelles results in the protection of the above enzyme from inactivation by the heparin-antithrombin complex. This sequestration of Factor $\mathrm{Xa}$ is not affected by the liberation of platelet release proteins or the molecular heterogeneity of the mucopolysaccharide preparations used. The magnitude of enzyme protection is strongly correlated with the extent of prothrombin activation at the time of heparin addition. On this basis, we suggest that high in vivo rates of thrombin generation may lead to the sequestration of Factor Xa on the platelet surface and hence allow this serine protease to resist the action of heparin until the complex is cleared from the circulation.
\end{abstract}

\section{INTRODUCTION}

The conversion of prothrombin to thrombin is mediated by the serine protease, Factor $\mathrm{Xa}$ and a cofactor, Factor $\mathrm{Va}$. The binding of Factor $\mathrm{Xa}$ to the platelet surface results in a 300,000 -fold augmentation in the ability of this enzyme to generate thrombin (1). This phenomenon has been referred to as the platelet procoagulant activity. Recent studies have revealed the emergence of $\sim 200$ high affinity binding sites for Factor $\mathrm{Xa}$ on the platelet surface when these cellular elements undergo the release reaction (1-3). Several independent lines of evidence suggest that platelet membrane-associated Factor Va serves as the receptor for

Address reprint requests to Dr. Robert D. Rosenberg.

Received for publication 23 June 1982 and in revised form 27 December 1982.
Factor $\mathrm{Xa}(1,4-8)$. The above data strongly indicate that the platelet surface must serve as an important site for thrombin formation. Prothrombin activation may also take place on phospholipid micelles (9-11). Under these conditions, this set of interactions leads to a 10,000-fold enhancement in the ability of Factor $\mathrm{Xa}$ to generate thrombin (9-11). However, the physiologic importance of the phospholipid surface as a locus for prothrombin conversion remains unclear.

Experimental data obtained in purified systems have demonstrated that the association of Factor Xa with the platelet surface or phospholipid micelles protects the enzyme against inhibition by the heparin-antithrombin complex $(1,12,13)$. Although these phenomena are of considerable significance with regard to the physiologic or pharmacologic action of the mucopolysaccharide, they have never been documented to occur within the complex milieu of whole blood.

In this communication, we have conducted a detailed examination of the accessibility of Factor Xa to neutralization by the heparin-antithrombin complex within plasma and whole blood. The above enzyme was detected by quantitating the concentrations of activation fragments $\left(F_{2} / F_{1+2}\right)$ cleaved by Factor $\mathrm{Xa}$ from prothrombin. The levels of $F_{2} / F_{1+2}$ were measured by means of a sensitive and specific radioimmunoassay (RIA) recently developed in our laboratory (14).

\section{METHODS}

Chemicals. Ionophore A23187 was purchased from Calbiochem-Behring Corp., American Hoechst Corp., San Diego, CA. Prostaglandin $I_{2}\left(\mathrm{PGI}_{2}\right)^{1}$ was kindly provided by Dr. Robert Handin, Brigham and Women's Hospital, Boston, MA.

All other chemicals were reagent grade or better.

Heparin. Porcine heparin (Panheprin) was bought from Abbott Laboratories, North Chicago, IL. Highly active hep-

\footnotetext{
${ }^{1}$ Abbreviation used in this paper: $\mathrm{PGI}_{2}$, prostaglandin $\mathbf{I}_{2}$.
} 
arins of mol wt 6,500 and 22,000 were prepared by Sephadex G-100 gel filtration and antithrombin-concanavalin A-Sepharose 4B affinity chromatography as previously described (15). The low molecular weight and high molecular weight species exhibited specific anticoagulant potencies of 350 U. S. Pharmacopoeia (USP) U/mg and 731 USP U/mg, respectively.

Lipid. Rabbit brain thromboplastin was obtained in 60 determination size vials from Ortho Diagnostics, Raritan, $\mathrm{NJ}$. The contents of each vial were suspended in $12 \mathrm{ml}$ of $0.15 \mathrm{M} \mathrm{NaCl}$ and then harvested by centrifugation at $4^{\circ} \mathrm{C}$ for $30 \mathrm{~min}$ at $25,000 \mathrm{~g}$. The entire process was repeated two additional times before suspending the washed rabbit brain thromboplastin in $12 \mathrm{ml}$ of $0.15 \mathrm{M} \mathrm{NaCl}$ just before use.

Proteins. Bovine serum albumin (RIA grade) was purchased from Sigma Chemical Company, St. Louis, MO. Rabbit immunoglobulin G (IgG) was provided by Miles Laboratories, Elkhart, IN. Hirudin was obtained from Pentapharm, Basel, Switzerland.

Measurement of protein concentration. Protein concentrations were determined by absorbance at $280 \mathrm{~nm}$. The molar extinction coefficients for $F_{2}$ and $F_{1+2}$ were assumed to be 12.5 and 12.9 , respectively (14).

Collection and processing of blood samples. Venipunctures were performed atraumatically with 21-gauge butterfly infusion sets by using a two-syringe technique. If anticoagulation was required, then blood was drawn into plastic syringes preloaded with the appropriate solutions as described below. These included: (a) $3.8 \%$ (wt/vol) sodium citrate; the ratio of anticoagulant to blood used was 1.0 to 9.0 (vol/vol). (b) EDTA, $10 \%$ (wt/vol) sodium ethylenediaminetetraacetic acid; the ratio of anticoagulant to blood used was 1.0 to 65.7 (vol/vol). (c) $\mathrm{F}_{2}$ anticoagulant, $7.3 \mathrm{mg} / \mathrm{ml}$ citric acid, $22 \mathrm{mg} /$ $\mathrm{ml}$ sodium citrate, $24.5 \mathrm{mg} / \mathrm{ml}$ dextrose, $2.234 \mathrm{mg} / \mathrm{ml}$ EDTA $1.602 \mathrm{mg} / \mathrm{ml}$ adenosine, and $25 \mathrm{U} / \mathrm{ml}$ heparin. The ratio of anticoagulant to blood used was 0.2 to 1.0 ( $\mathrm{vol} / \mathrm{vol}$ ). Plateletrich plasma was obtained by centrifugation of anticoagulated blood at $20^{\circ} \mathrm{C}$ for $10 \mathrm{~min}$ at $400 \mathrm{~g}$. Platelet counts were estimated by hemocytometer by using phase-contrast microscopy. Platelet-poor plasma was obtained by centrifugation of anticoagulated blood at $4^{\circ} \mathrm{C}$ for $20 \mathrm{~min}$ at $1,600 \mathrm{~g}$.

RIA of $F_{2} / F_{1+2}$. The plasma levels of $F_{2} / F_{1+2}$ were quantitated by a double-antibody RIA that is completely specific for these activation fragments (14). To this end, $50 \mu \mathrm{l}$ of seven different concentrations of $F_{1+2}$ standards or plasma samples or RIA buffer (see below) was added in duplicate to $50 \mu \mathrm{l}$ of ${ }^{125} \mathrm{I}-\mathrm{F}_{1+2}(\sim 5,000 \mathrm{cpm})$ and $100 \mu \mathrm{l}$ of the specific $F_{2} / F_{1+2}$ antibody population. The dilution of labeled $F_{1+2}$ and the antibody population was carried out with $0.155 \mathrm{M}$ $\mathrm{NaCl}$ and $0.05 \mathrm{M}$ EDTA in $0.0255 \mathrm{M}$ sodium phosphate, $\mathrm{pH}$ 7.4 with $0.6 \%$ (wt/vol) sodium azide and $3.0 \%$ (wt/vol) bovine serum albumin added (RIA buffer). The dilution of the $F_{1+2}$ standards and plasma samples was conducted with human plasma adsorbed with barium chloride and extensively dialyzed against RIA buffer. The concentration of specific $F_{2} / F_{1+2}$ antibody population had been predetermined to precipitate $25-33 \%$ of the radiolabeled $F_{1+2}$ in the absence of competing antigen. After incubation of the various mixtures at $4^{\circ} \mathrm{C}$ for $18 \mathrm{~h}$, the separation of bound from free radiolabeled antigen was accomplished by the second antibody technique. For this purpose, $50 \mu \mathrm{l}$ of rabbit IgG and $200 \mu$ l of sheep anti-rabbit antisera were both added to each tube. The amount of rabbit IgG used was predetermined to result in maximal precipitation with $200 \mu \mathrm{l}$ of the sheep antirabbit antisera. After incubation at $4^{\circ} \mathrm{C}$ for $18 \mathrm{~h}$, the tubes were centrifuged at $4^{\circ} \mathrm{C}$ for $10 \mathrm{~min}$ at $6,450 \mathrm{~g}$ and subsequently washed three times at $4^{\circ} \mathrm{C}$ with $0.2 \mathrm{ml}$ of RIA buffer modified by the addition of only $0.2 \%$ (wt/vol) bovine serum albumin. The resultant precipitates were then quantitated for ${ }^{125}$ I counts.

RIA of PF-4. Measurement of PF-4 was performed by Dr. Robert I. Handin, Boston, MA, as previously outlined (16).

Analysis of RIA data. Dose-response curves were constructed by using a computer-assisted least squares fit of the raw data to the four-parameter model of Rodbard et al. (17, 18). Statistical analyses of data were conducted by standard techniques (19).

\section{RESULTS}

Antithrombin is the principal antagonist of Factor Xa. In the presence of heparin, the interaction of these two proteins is almost instantaneous (20). Therefore, the addition of mucopolysaccharide to the blood should suppress Factor Xa-induced cleavage of prothrombin with resultant inhibition of $F_{2} / F_{1+2}$ generation. The continuing elaboration of activation fragments under the above conditions would indicate the inaccessibility of Factor $\mathrm{Xa}$ to the heparin-antithrombin complex.

The previous set of considerations prompted us to examine the heparin inhibition of Factor Xa within the complex milieu of whole blood. To this end, blood was collected without anticoagulant, placed in a 50$\mathrm{ml}$ conical tube at $24^{\circ} \mathrm{C}$, and stirred continuously in a gentle fashion. Clot formation usually occurred at $\mathrm{F}_{1+2}$ levels of $>100 \mathrm{nM}$. However, the fibrin meshwork was localized to the stirring magnet and did not interfere with subsequent sampling. At varying times, aliquots were removed from the above mixture and mucopolysaccharide was added at a final concentration of $2 \mathrm{U} / \mathrm{ml}$. Each of the aliquots were sampled on four to five occasions over the next 15-20 min and the resultant solutions were immediately mixed with a specific $F_{2}$ anticoagulant to suppress the further cleavage of prothrombin. We have previously demonstrated that scission of the zymogen within blood by concentrations of Factor Xa as high as $5 \mu \mathrm{g} / \mathrm{ml}$ is completely inhibited by this treatment (21). Plasma was obtained from the above solutions by centrifugation as described in Methods and the concentrations of $F_{2} / F_{1+2}$ were estimated by RIA. The presence of $F_{1+2}$ reflects the Factor Xa-dependent cleavage of prothrombin at $\mathrm{Arg}_{273^{-}}$ $\mathrm{Thr}_{274}$. The $\mathrm{F}_{1+2}$ fragment is then clipped at $\mathrm{Arg}_{155^{-}}$ $\mathrm{Ser}_{156}$ by Factor $\mathrm{Xa}$ or thrombin, which is responsible for the liberation of $F_{1}$ and $F_{2}$. No attempt was made to individually quantitate $F_{2}$ or $F_{1+2}$, since both fragments have identical immunoreactivities in our RIA (21).

The results of a typical experiment are summarized in Fig. 1. Aliquots were removed from the stirred blood at 10.5, 12.5, 13.5, and $20 \mathrm{~min}$ of incubation and the solutions were immediately mixed with mucopolysaccharide. Samples were obtained from the initial three separate aliquots of heparinized blood over the subsequent 16-20 min and the concentrations of $F_{2} / F_{1+2}$ were quantitated as a function of time. Data presented 


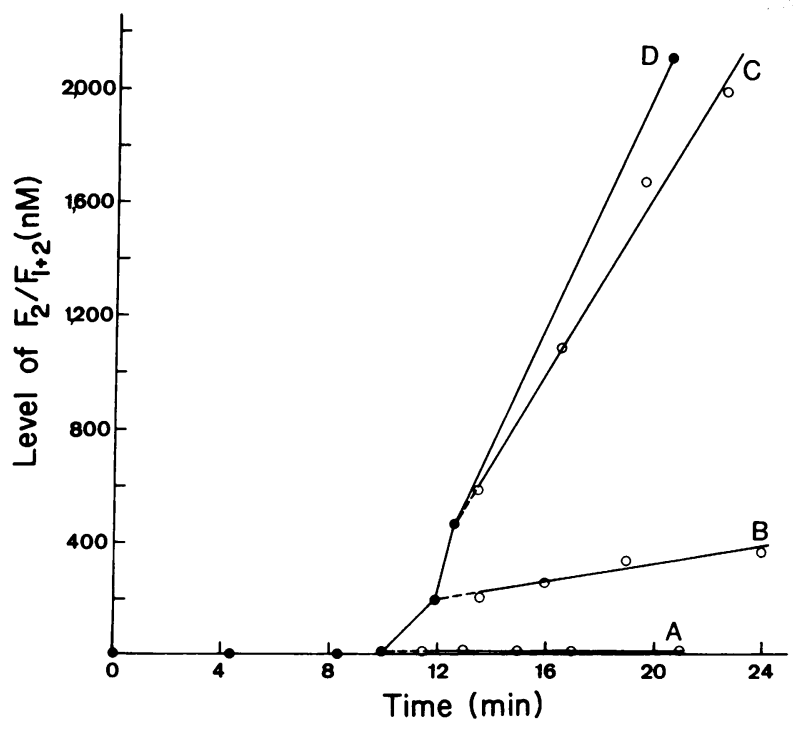

Figure 1 The accessibility of Factor Xa to the heparin-antithrombin complex within whole blood. The concentrations of $F_{2} / F_{1+2}(\bullet)$ were determined at varying times within nonanticoagulated whole blood gently stirred at $24^{\circ} \mathrm{C}$ (curve D). Aliquots were removed at three different levels of zymogen conversion and immediately mixed with heparin at a final concentration of $2 \mathrm{U} / \mathrm{ml}$. The levels of $\mathrm{F}_{2} / \mathrm{F}_{1+2}(\mathrm{O})$ were quantitated at varying times after addition of mucopolysaccharide. The lines represent the best linear least squares fit of the above data. See text and Methods for additional experimental details.

in Fig. 1 suggest that the degree to which Factor $\mathrm{Xa}$ can be neutralized is dependent upon the extent of thrombin production at the time of mucopolysaccharide addition. At low levels of prothrombin conversion $(\sim 0.1 \%)$, the serine protease is completely inhibited by the heparin-antithrombin complex (curve A), whereas at higher levels of zymogen transformation (10-25\%), the above enzyme is less accessible to the mucopolysaccharide-protease inhibitor complex (curves $B$ and $C$ ). Similar results have been obtained when heparin is added to blood at final concentrations as high as $50 \mathrm{U} / \mathrm{ml}$.

The quantitative nature of this relationship was examined by computing the linear least square slopes of the $F_{2} / F_{1+2}$ generation curves at varying initial concentrations of activation fragments for 20 separate experiments in which heparin was used at a final concentration of $2 \mathrm{U} / \mathrm{ml}$ (data not shown). The individual curves exhibited considerable linearity as suggested by the mean linear least squares correlation coefficient $(r)$ of $0.924 \pm 0.113$ (SD). As expected, this characteristic behavior is absent when the levels of prothrombin have been sufficiently depleted to reduce $F_{2} / F_{1+2}$ generation over the next $12-20 \mathrm{~min}$. For this reason, individual curves in which the initial concentration of $\mathrm{F}_{2} / \mathrm{F}_{1+2}$ exceeded $2,000 \mathrm{nM}$ were excluded from consideration. In Fig. 2, we have plotted the calculated slopes of the $F_{2} / F_{1+2}$ generation curves vs. the initial concentrations of activation fragment at the time of mucopolysaccharide addition. Linear least squares analysis revealed that the above data is best represented by the equation $y=0.138 x+13.4$, with $r$ $=0.903$. This relationship suggests that the protection of Factor $\mathrm{Xa}$ from the heparin-antithrombin complex is a linear function of the extent of prothrombin conversion.

It might be argued that the rate of neutralization of the above enzyme by the mucopolysaccharide-protease inhibitor complex is actually dependent upon the period of time from the onset of prothrombin activation to the addition of heparin. If this were the case, an apparent relationship would exist between Factor $\mathrm{Xa}$ accessibility and fractional zymogen conversion. To test this hypothesis, 16 experiments were selected for analysis in which the available $F_{2} / F_{1+2}$ measurements permitted an extrapolated estimate of the time of onset of prothrombin activation. The slopes of the $F_{2} / F_{1+2}$ generation curves were then plotted vs. the time interval from initiation of zymogen transformation to the admixture of mucopolysaccharide which ranged from 2.6 to $13 \mathrm{~min}$. Linear least squares analyses of the two parameters yielded an $r$ value $=0.716$, which is considerably smaller than that observed earlier. This finding suggests that the accessibility of Factor $\mathrm{Xa}$ to the heparin-antithrombin complex is a linear function of the extent of prothrombin activation rather than the time interval from the onset of zymogen conversion to the addition of mucopolysaccharide.

The experiments cited above were conducted with commercial preparations of heparin. These products are known to contain mucopolysaccharide species with mol wt that range from 3,000 to $35,000(22,23)$. Furthermore, only $\sim 30 \%$ of heparin components of any given molecular size possess the unique monosaccharide sequences required for anticoagulant function $(23,24)$. To exclude the possibility that the relationship between Factor $\mathrm{Xa}$ accessibility and fractional zymogen conversion was mediated by a particular anticoagulant or nonanticoagulant fraction of the commercial preparation, the experiments outlined above were repeated with highly active heparins of mol wt 6,500 and 22,000 , respectively. In Fig. 2, we have plotted the slopes of the $F_{2} / F_{1+2}$ generation curves obtained with the two homogenous mucopolysaccharide products vs. the initial concentrations of activation fragments at the time of heparin addition. Linear least squares analyses demonstrated that the above data is best represented by the equation $y=0.10 x+21.7$, with $r=0.916$ (not shown). This data is virtually identical to that obtained with commerical preparations of the mucopolysaccharide.

Previous investigators have partially defined the molecular events that result in the binding of Factor 


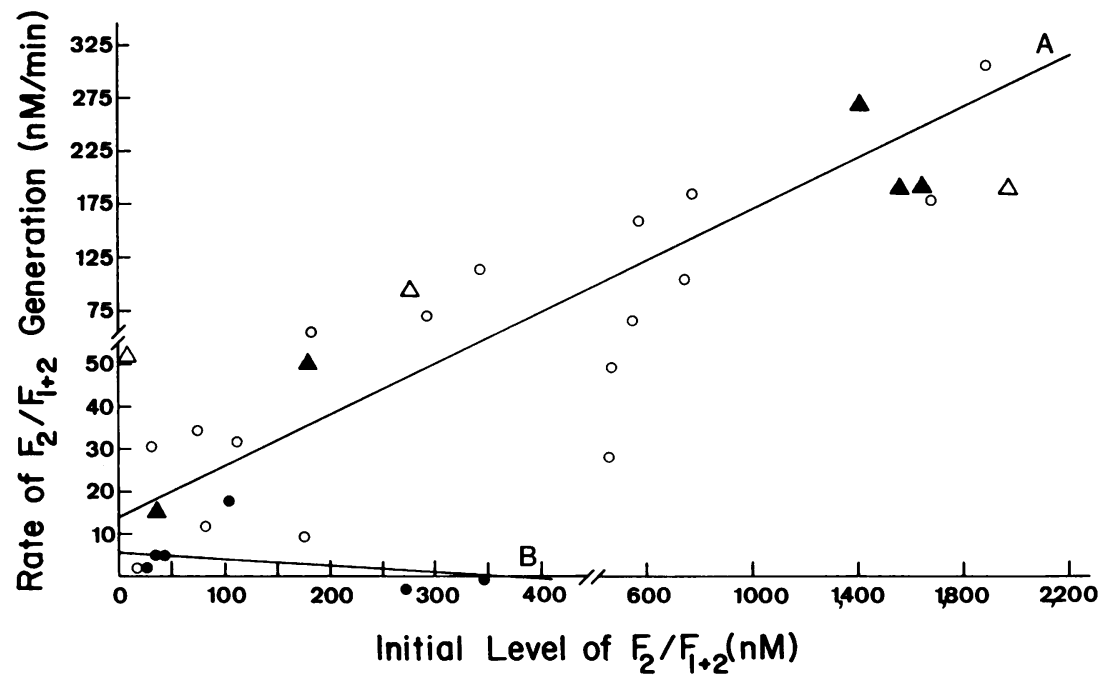

FIGURE 2 The extent of Factor Xa protection from neutralization by the heparin-antithrombin complex within whole blood in the presence and absence of $\mathbf{P G I}_{2}$. The concentrations of $\mathbf{F}_{2} /$ $F_{1+2}$ were determined at varying times within nonanticoagulated blood in the presence and absence of $\mathrm{PGI}_{2}$. Aliquots were removed at different levels of zymogen conversion and immediately mixed with commercial heparin or highly active heparin of mol wt 22,000 or highly active heparin of mol wt 6,500 at final concentrations of $2 \mathrm{U} / \mathrm{ml}$. The levels of $\mathrm{F}_{2} / \mathrm{F}_{1+2}$ were quantitated at intervals of $4 \mathrm{~min}$ over the next 16-20 min after addition of mucopolysaccharide. The slopes of the $F_{2} / F_{1+2}$ generation curves were determined by linear least squares analyses. The rates of $F_{2} / F_{1+2}$ production in the presence of commercial heparin with $(\bullet)$ or without (O) $\mathrm{PGI}_{2}$ as well as the rates of $\mathrm{F}_{2} / \mathrm{F}_{1+2}$ production in the presence of highly active heparin of mol wt 22,000 $(\Delta)$ and 6,500 $(\Delta)$ are plotted against the concentrations of activation fragments before heparin addition. The solid lines represent the best linear squares fit of the data obtained for commercial heparin in the presence and absence of $\mathrm{PGI}_{2}$. See text and Methods for additional experimental details.

$\mathrm{Xa}$ to the platelet surface $(1,4-8)$. These studies indicate that the generation of thrombin is responsible for the transformation of Factor $\mathrm{V}$ to Factor $\mathrm{Va}$ as well as the induction of the platelet release reaction. Factor $\mathrm{Va}$ is then able to interact with a specific region on the surface of the "activated" platelets and the resultant protein-membrane complex represents the high affinity receptor for Factor Xa. If protection of this serine protease from neutralization by the heparin-antithrombin complex required formation of the above receptor, then suppression of the platelet release reaction should permit the free or weakly bound enzyme to be inactivated by the mucopolysaccharide-protease inhibitor complex. In this case, no linear relationship would be observed between Factor Xa accessibility and fractional zymogen conversion.

To test this hypothesis, $\mathrm{PGI}_{2}$, a potent inhibitor of the platelet release reaction, was added to nonanticoagulated whole blood at a final concentration of 15 $\mu \mathrm{M}$. Subsequently, aliquots were removed at differing levels of prothrombin activation, immediately mixed with heparin, and the concentrations of $F_{2} / F_{1+2}$ were quantitated as a function of time. Given the relatively short half-life of $\mathrm{PGI}_{2}$, constant levels of the prostaglandin were maintained by repetitive addition at in- tervals of $3 \mathrm{~min}$. In all cases, the suppression of the platelet release reaction was documented by RIA for $\mathrm{PF}-4$. The mean concentration of this release protein within the fluid phase of blood averaged $18.2 \mathrm{ng} /$ $\mathrm{ml} \pm 8.5$ (SD), $n=8$. This value is $\leq 11 \%$ of the PF-4 levels measured in nine other experiments that were conducted in the absence of $\mathbf{P G I}_{2}$.

It should be noted that significant zymogen conversion is difficult to achieve in the presence of the above prostaglandin. This is not surprising in view of our previous demonstration of the relationship between platelet secretion and prothrombin activation (16). However, a significant degree of zymogen transformation occurred in several experiments before the addition of heparin. In Fig. 2, we have plotted the slopes of the $F_{2} / F_{1+2}$ generation curves obtained from the above experiments vs. the initial concentrations of activation fragments. The data is best represented by the nearly horizontal line described by the equation $y$ $=0.014 x+5.56$, with $r=0.246$. Thus, the linear relationship between Factor Xa accessibility and fractional zymogen conversion no longer holds in the absence of platelet secretion.

It should be noted that several experiments were not included in the above analyses. In these instances, in- 
cubation of the nonanticoagulated blood was sufficiently prolonged to permit $F_{2} / F_{1+2}$ levels to exceed $440 \mathrm{nM}$ (conversion of $>15 \%$ of the plasma prothrombin). Under these conditions, the extent of Factor Xa protection was quite variable. This inconsistency is most probably due to the prolonged exposure of platelets to significant levels of thrombin with subsequent nonspecific proteolysis of cellular elements.

The results obtained with $\mathrm{PGI}_{2}$ addition to nonanticoagulated blood do not exclude the hypothesis that protection of Factor Xa from the heparin-antithrombin complex is mediated by a soluble factor secreted during the platelet release reaction. This substance might impair the binding of mucopolysaccharide to protease inhibitor or the interaction between Factor $\mathrm{Xa}$ and the heparin-antithrombin complex. The preceding supposition appears unlikely in view of the fact that the linear relationship depicted in Fig. 2 is valid at mucopolysaccharide concentrations as high as $50 \mathrm{U} /$ $\mathrm{ml}$ (see above). However, the presence of the postulated species was directly sought by inducing platelets to undergo the release reaction and then using the platelet-poor plasma to quantitate Factor Xa accessibility to the heparin-antithrombin complex.

To this end, platelet-rich plasma was prepared from blood anticoagulated with EDTA as described in Methods. Platelet counts ranged from $339 \times 10^{9}$ to 534 $\times 10^{9}$ cells/liter. The above cellular elements were induced to undergo the release reaction by addition of Ionophore A23187 at a final concentration of $10 \mu \mathrm{M}$. The successful activation of platelets was confirmed by establishing that the resultant levels of PF-4 within the fluid phase of blood were not significantly different from those obtained during the clotting of nonanticoagulated blood $(P<0.5)$. The aggregated cellular elements were then removed by centrifugation at $4^{\circ} \mathrm{C}$ for $20 \mathrm{~min}$ at $1,600 \mathrm{~g}$. The coagulation process was initiated within the platelet-poor plasma by addition of washed rabbit brain thromboplastin and calcium chloride at final concentrations of $15 \mu \mathrm{g} / \mathrm{ml}$ and 7.5 $\mathrm{mM}$, respectively. Aliquots were removed from the above incubation mixture at differing levels of zymogen conversion, heparin was added to each sample at a final concentration of $2 \mathrm{U} / \mathrm{ml}$, and the generation of $\mathrm{F}_{2} / \mathrm{F}_{1+2}$ was determined as a function of time.

The linear least squares slopes from nine such experiments were plotted vs. the initial levels of activation fragment at the time of mucopolysaccharide addition. The data (not shown) is best described by the equation $y=0.039 x+4.68$, with $r=0.488$. The shallow slope and poor correlation between the rates of prothrombin conversion and the concentrations of $F_{2} / F_{1+2}$ at the time of heparin admixture suggest that protection of Factor $\mathrm{Xa}$ is minimal in the above milieu. Thus, it is unlikely that a substance which is secreted by platelets is responsible for the relationship between
Factor Xa accessibility and fractional zymogen conversion.

The essential role of platelets in the protection of Factor Xa from inactivation by the heparin-antithrombin complex was confirmed by experiments conducted with platelet-rich plasma and platelet-poor plasma. To this end, platelet-rich plasma was prepared from blood anticoagulated with EDTA as described in Methods. Platelet counts ranged from $339 \times 10^{9}$ to $534 \times 10^{9}$ cells/liter. These cellular elements were induced to undergo the release reaction by addition of Ionophore A23187 at a final concentration of $10 \mu \mathrm{M}$. The activation of platelets was confirmed by demonstrating that the resultant levels of PF-4 within the fluid phase were not significantly different from those obtained during the clotting of nonanticoagulated blood $(P$ $<0.5$ ). After the above mixture was incubated at $24^{\circ} \mathrm{C}$ for 5 min, calcium chloride was added at a final concentration of $7.5 \mathrm{mM}$ and aliquots were removed at differing levels of zymogen conversion. The various solutions were immediately mixed with heparin at a final concentration of $2 \mathrm{U} / \mathrm{ml}$ and the levels of $F_{2} / F_{1+2}$ were determined at intervals of 4 min during the next $16 \mathrm{~min}$. The results of a typical experiment are shown in Fig. 3A. Two aliquots were removed from the activation mixtures at initial $F_{2} / F_{1+2}$ concentrations of $33.6 \mathrm{nM}$ and $452 \mathrm{nM}$, respectively. The slopes of the $F_{2} / F_{1+2}$ generation curves for the above samples after addition of mucopolysaccharide were $7.3 \mathrm{nM} / \mathrm{min}$ and $79.5 \mathrm{nM} / \mathrm{min}$, respectively. The steeper slope of the $F_{2} / F_{1+2}$ generation curve at a higher initial concentration of activation fragment suggests that Factor Xa may be protected from neutralization by the heparinantithrombin complex under the above conditions.

In parallel experiments, we also examined Factor $\mathrm{Xa}$ accessibility to the mucopolysaccharide-protease inhibitor complex within platelet poor-plasma. To this end, platelet-poor plasma was obtained from blood anticoagulated with EDTA as described in Methods. The coagulation process was initiated at $24^{\circ} \mathrm{C}$ by addition of washed rabbit brain thromboplastin and calcium chloride at final concentrations of $15 \mu \mathrm{g} / \mathrm{ml}$ and $7.5 \mathrm{mM}$, respectively. Aliquots were removed from the above incubation mixture at differing levels of zymogen conversion, heparin was added to each sample at a final concentration of $2 \mathrm{U} / \mathrm{ml}$, and the levels of $F_{2} / F_{1+2}$ were determined within each solution at intervals of 4 min during the next $16 \mathrm{~min}$. The results of a typical experiment are shown in Fig. 3B. Two aliquots were removed from the activation mixture at initial $F_{2} / F_{1+2}$ concentrations of $271 \mathrm{nM}$ and $1,022 \mathrm{nM}$, repectively. The slopes of the $F_{2} / F_{1+2}$ generation curves for the above samples, after addition of mucopolysaccharide were $34.3 \mathrm{nM} / \mathrm{min}$ and $19.5 \mathrm{nM} /$ min, respectively. The relatively constant but shallow slopes of the $F_{2} / F_{1+2}$ generation curves at two widely 


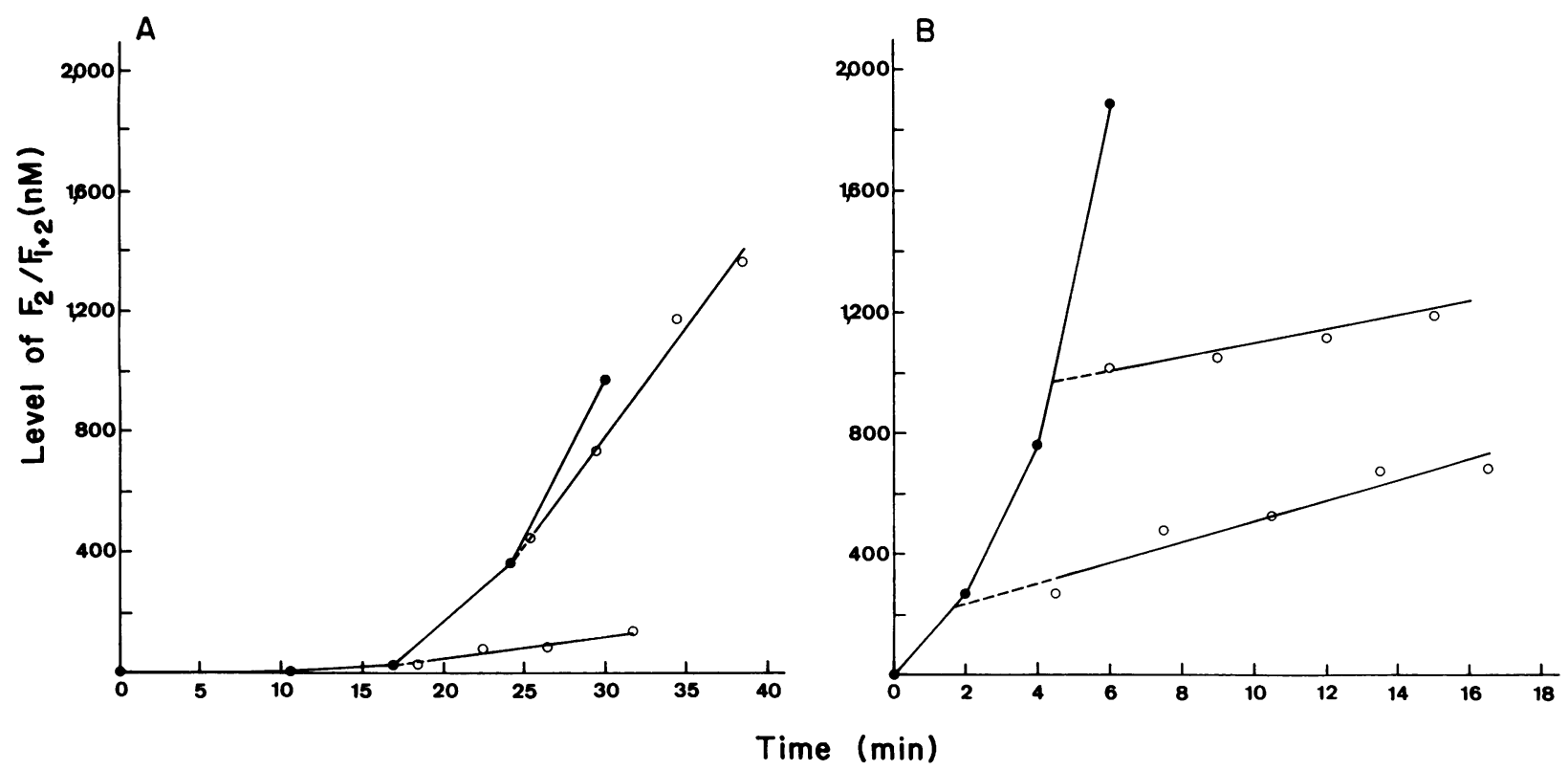

Figure 3 The accessibility of Factor $\mathrm{Xa}$ to neutralization by the heparin-antithrombin complex within platelet-rich plasma and platelet-poor plasma. $A$, The concentration of $F_{2} / F_{1+2}(\bullet)$ was determined at varying times within platelet-rich plasma. The coagulation process was initiated with ionophore 23187 and subsequent addition of calcium chloride. Aliquots were removed at two different levels of zymogen conversion and immediately mixed with heparin at a final concentration of $2 \mathrm{U} / \mathrm{ml}$. The levels of $\mathrm{F}_{2} / \mathrm{F}_{1+2}(\mathrm{O})$ were quantitated at varying times after admixture of mucopolysaccharide. The lines represent the best linear fit of the above data. B, The concentrations of $\mathrm{F}_{2} / \mathrm{F}_{1+2}(\bullet)$ were determined at varying times within platelet-poor plasma. The coagulation process was initiated by the addition of rabbit brain thromboplastin and calcium chloride. Aliquots were removed at two different levels of zymogen conversion and immediately mixed with heparin at a final concentration of $2 \mathrm{U} / \mathrm{ml}$. The levels of $F_{2} / F_{1+2}$ $(O)$ were quantitated at varying times after admixture of mucopolysaccharide. The lines represent the best linear fit of the above data.

different initial levels of activation fragment suggest that Factor $\mathrm{Xa}$ is not protected by phospholipid from neutralization by the heparin-antithrombin complex under the above conditions.

The extent of Factor Xa accessibility to the heparinantithrombin complex was examined in greater detail by conducting 34 additional experiments within platelet-rich plasma and platelet-poor plasma as outlined above. In each instance, the linear least squares slopes of $F_{2} / F_{1+2}$ production after mucopolysaccharide addition were computed as previously described. Fig. 4 depicts a plot of the slopes of the $F_{2} / F_{1+2}$ generation curves as a function of the initial concentrations of total activation fragment at the time of heparin addition. Data obtained for platelet-rich plasma and platelet-poor plasma are best described by the equations $y=0.091 x+29.7$, with $r=0.776$; and $y=0.008$ $x+9.16$, with $r=0.338$, respectively. The slopes of the two analytic expressions clearly indicate that platelets but not phospholipids are capable of protecting Factor Xa against neutralization by the heparin-antithrombin complex.

\section{DISCUSSION}

Several groups have examined the accessibility of Factor $\mathrm{Xa}$ to neutralization by the heparin-antithrombin complex in the presence of phospholipid micelles or activated platelets. The studies of Yin (25), Marciniak (12), as well as Walker and Esmon (13) conducted with purified components have shown that phospholipid with or without Factor $\mathrm{Va}$ and calcium ions can protect Factor Xa against inactivation by the mucopolysaccharide-protease inhibitor complex. The investigations of Miletich et al. (1) carried out with purified proteins and washed, thrombin activated platelets suggest that the binding of Factor Xa to the above cellular surface via Factor Va renders this serine protease inaccessible to the action of the heparin-antithrombin complex. However, it is not clear that either of these mechanisms operate within complex physiologic fluids.

We have examined the neutralization of Factor $\mathrm{Xa}$ by the heparin-antithrombin complex within whole human blood. This analysis was conducted with a specific RIA that measures the levels of $F_{2} / F_{1+2}$ generated 


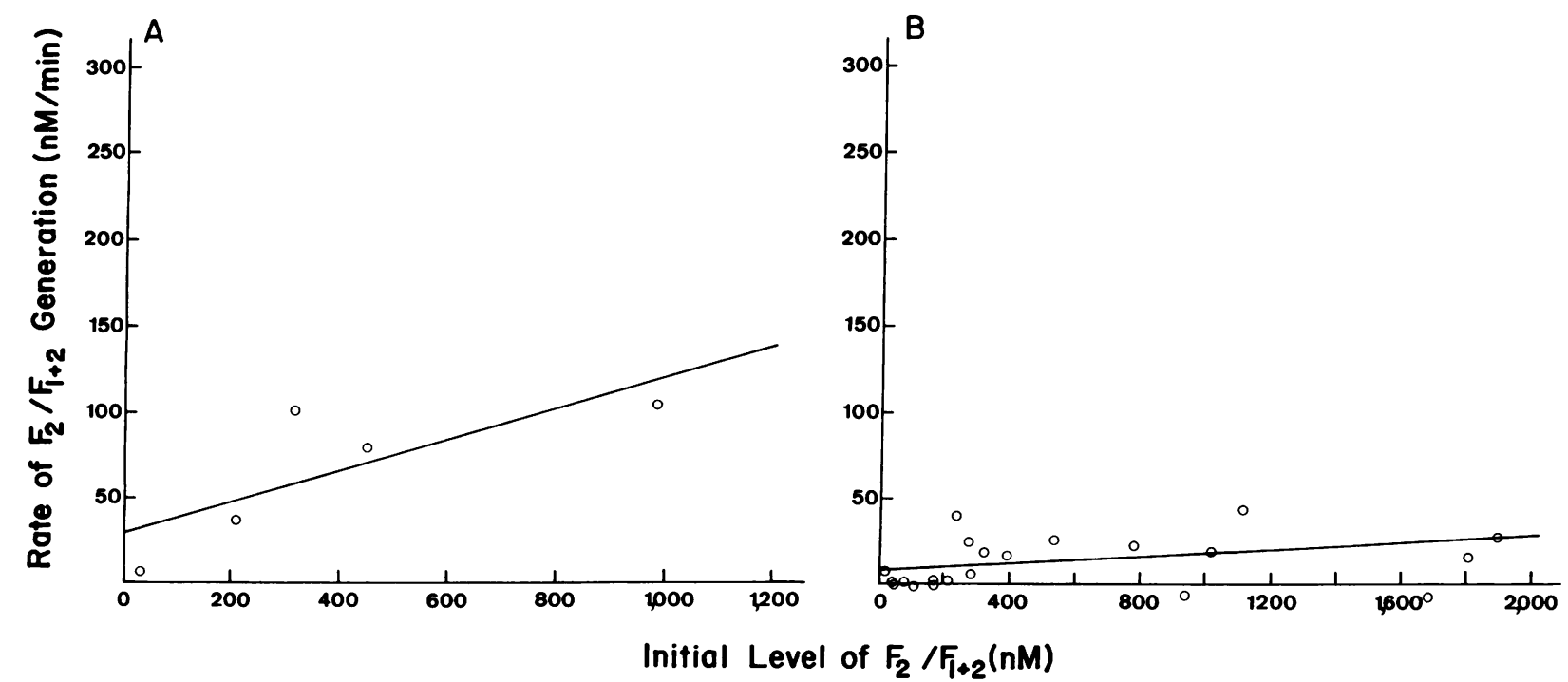

Figure 4 The extent of Factor Xa protection from neutralization by the heparin-antithrombin complex within platelet-rich plasma and platelet-poor plasma. A, The concentrations of $F_{2} /$ $F_{1+2}$ were determined at varying times after initiation of the coagulation process within plateletrich plasma. Aliquots were removed at different levels of zymogen conversion and immediately mixed with heparin at a final concentration of $2 \mathrm{U} / \mathrm{ml}$. The rate of $\mathrm{F}_{2} / \mathrm{F}_{1+2}$ production (O) were determined over the next 16-20 min after addition of mucopolysaccharide and are plotted against the concentrations of activation fragments before heparin admixture. $B$, The concentrations of $F_{2} / F_{1+2}$ were determined at varying times after initiation of the coagulation process within platelet-poor plasma. Aliquots were removed at different levels of zymogen conversion and immediately mixed with heparin at a final concentration of $2 \mathrm{U} / \mathrm{ml}$. The rates of $\mathrm{F}_{2} / \mathrm{F}_{1+2}$ production $(O)$ were determined over the next 16-20 min after addition of mucopolysaccharide and are plotted against the concentrations of activation fragments before heparin admixture. The lines represent the best linear least squares fit of the data obtained for platelet-rich plasma and platelet-poor plasma. See text and Methods for additional experimental details.

during the initial Factor Xa-dependent cleavage of prothrombin. Our data reveal that protection of Factor $\mathrm{Xa}$ from inactivation by the mucopolysaccharide-protease inhibitor complex is not absolute in nature but is a linear function of the extent of zymogen conversion. ${ }^{2}$ The above relationship is critically dependent upon the ability of platelets to undergo the release reaction. This was established by monitoring the neutralization of Factor $\mathrm{Xa}$ by the heparin-antithrombin complex within whole human blood, while suppressing platelet secretion by addition of $\mathrm{PGI}_{2}$. Our results demonstrate that Factor $\mathrm{Xa}$ is completely accessible to inactivation by the mucopolysaccharide-protease inhibitor complex at all levels of zymogen conversion under the above conditions.

Furthermore, we have also compared the accessibility of Factor Xa to the heparin-antithrombin complex within platelet-rich and platelet-poor plasma. Prothrombin conversion was initiated within platelet-

${ }^{2}$ We have assumed that contributions to Factor Xa levels via a heparin-resistant pathway are minimal. This appears reasonable since activation of this zymogen by Factor IXa is also sensitive to the action of the mucopolysaccharide. rich plasma by exposing these cellular elements to ionophore and then adding calcium ions. Generation of thrombin within platelet-poor plasma was started by admixture of rabbit brain thromboplastin and calcium ions. Our data show that Factor Xa protection from the heparin-antithrombin complex is a linear function of the extent of zymogen conversion within plateletrich plasma but that this relationship is not apparent within platelet-poor plasma. These findings not only confirm the importance of the platelet release reaction in the sequestration of the above enzyme but also indicate that phospholipids are unable to perform this function within human plasma.

We have considered the possibility that the relationship between Factor Xa accessibility and fractional zymogen conversion might be due to a progressive release of heparin-neutralizing factors from the platelet or to the inactive mucopolysaccharide within commercial preparations of the anticoagulant.

To this end, platelet-rich plasma was exposed to concentrations of ionophore that induced these cellular elements to undergo the release reaction after removal of aggregated platelets. The coagulation process was initiated by addition of rabbit brain thromboplastin 
and calcium ions in the presence of the same concentration of heparin previously used. The neutralization of Factor Xa was no different within this system from that observed with platelet-poor plasma in which the release reaction had not taken place.

Furthermore, we have also analyzed the inhibition of Factor Xa during in vitro coagulation of whole blood in the presence of highly active heparin of mol wt $\sim 6,500$ and 22,000 , respectively. No difference in the protection of the enzyme was noted with the above products as compared with commercial preparations of mucopolysaccharide.

Previous investigations carried out with purified components suggest that thrombin generation within the blood would result in the transformation of Factor $\mathrm{V}$ to Factor $\mathrm{Va}$ as well as the induction of the platelet release reaction $(1-6,8)$. Under these conditions, one would expect Factor $\mathrm{Xa}$ to bind tightly to the surface of the activated platelet via Factor $\mathrm{Va}$ and thereby become inaccessible to the action of the heparin-antithrombin complex within blood $(1,12,13)$. The experimental data cited here indicate that protection of Factor Xa from neutralization by the mucopolysaccharide-protease inhibitor complex during in vitro coagulation of whole human blood is completely dependent upon the presence of activated platelets. These results strengthened our belief that the Factor Xa protective mechanism observed with purified components is relevant to complex physiologic fluids. However, it is surprising that Factor $\mathrm{Xa}$ accessibility to the heparinantithrombin complex is not absolute in nature but is strongly correlated with fractional zymogen conversion. In principle, this unanticipated observation might arise from a linear relationship between the degree of the platelet release reaction or the level of Factor $\mathrm{Xa}$ generation or the extent of Factor $\mathrm{V}$ to Factor Va transformation and the amount of thrombin generated within the blood. Alternatively, the activation of platelets and the production of Factor $\mathrm{Va}$ and Factor $\mathrm{Xa}$ might take place at levels insufficient to form the prothrombinase complex during the earlier stages of in vitro coagulation of whole blood. However, the subsequent time-dependent association of the three components might be mistaken for a relationship between Factor Xa accessibility and fractional zymogen conversion.

We have considered the possibility that a progressive increase in the degree of the platelet release reaction might be responsible for our observations. On the one hand, in preliminary experiments, we have incubated platelet-rich plasma with ionophore to induce secretion as documented by RIA for PF-4. This mixture was then recalcified and the accessibility of Factor $\mathrm{Xa}$ to the heparin-antithrombin complex was determined. No difference was noted in the expected linear relationship between Factor Xa protection and the extent of zymogen conversion (data not shown). On the other hand, we have previously demonstrated that during in vitro coagulation of whole blood, a significant number of platelets undergo the release reaction within the initial phase of prothrombin transformation (16). Thus, it would be surprising if limiting numbers of activated platelets represented the basic phenomena underlying the observed dependence.

It also seems somewhat unlikely that the time-dependent assembly of the prothrombinase complex on the surface of the platelet could be responsible for the observed linkage between Factor Xa accessibility and fractional zymogen conversion. Although it is difficult to unambiguously assess this possibility, our data suggest that the rates of Factor $\mathrm{Va}$ and Factor $\mathrm{Xa}$ interaction with activated platelets are not involved in generating the above relationship. The transformation of prothrombin within whole blood begins at variable intervals after venipuncture $(2.6-13 \mathrm{~min})$. If the essential components of the prothrombinase complex formed early in the coagulation process and then proceeded to bind in a progressive fashion to the platelet surface, protection of Factor $\mathrm{Xa}$ would be more strongly correlated with the time interval from the onset of $F_{2} / F_{1+2}$ generation to the point of heparin addition rather than with the extent of zymogen conversion. Careful analyses of our observations reveal that the former relationship is statistically weaker than the latter one.

Our experimental data do not permit us to draw definitive conclusions concerning the underlying molecular interactions that are ultimately responsible for the linear relationship between Factor Xa accessibility and fractional zymogen conversion. However, the following scenario of events appears a possible cause of our intriguing observation. Initially, thrombin generation within the blood leads to the transformation of small amounts of Factor $\mathrm{V}$ to Factor $\mathrm{Va}$ as a linear function of enzyme production. The latter phenomenon is possibly due to the presence of other thrombin substrates within blood at much higher concentrations than Factor V. Subsequently, the increasing levels of Factor $\mathrm{Va}$ are responsible for a growing number of Factor Xa molecules that are tightly bound to the surface of the activated platelet and hence, directly result in an enhanced sequestration of the latter enzyme from the action of the heparin-antithrombin complex.

It should be noted that the observed correlation between Factor Xa accessibility and fractional zymogen conversion may have important implications with respect to the treatment of thrombotic disease. The relationship outlined above would predict that high in vivo rates of thrombin generation might lead to the formation of substantial amounts of activated plateletFactor Va-Factor $\mathrm{Xa}$ interaction product. The sequestered Factor $\mathrm{Xa}$ hidden within this multimole- 
cular complex should be resistant to the action of heparin and therefore must be cleared from the circulation before anticoagulant cover can be safely withdrawn. We have recently studied two patients with disseminated intravascular coagulation who exhibited grossly elevated concentrations of $F_{2} / F_{1+2}$, thrombin-antithrombin complex and fibrinopeptide A. Treatment of these individuals with full doses of heparin for $24 \mathrm{~h}$ or longer resulted in a dramatic lowering of fibrinopeptide A levels towards the normal range without any concomitant reduction of $\mathrm{F}_{2} / \mathrm{F}_{1+2}$ and thrombin-antithrombin complex levels. We suspect that these patients may have significant amounts of Factor Xa bound to activated platelets, which cannot be neutralized by heparin-antithrombin interaction products. In this case, the above sequestered enzyme would generate thrombin that is prevented from acting on fibrinogen by the mucopolysaccharide-protease inhibitor complex. If this supposition is correct, the activated platelet-Factor Va-Factor $\mathrm{Xa}$ interaction product must be cleared from the blood before treatment with heparin can be halted without renewed formation of fibrin thrombi. The above situation may represent a general phenomenon in which the duration of adequate heparin therapy is partially determined by the intensity of the thrombotic stimulus via the generation of activated platelet-Factor Va-Factor Xa complex.

\section{ACKNOWLEDGMENTS}

This research was supported in part by National Institutes of Health grants HL 28625, HL 28960, HL 25066, CA 19589; National Foundation for Cancer Research, Medical Research Council of Canada, and American Heart Association.

\section{REFERENCES}

1. Miletich, J. P., C. M. Jackson, and P. W. Majerus. 1978. Properties of the factor $\mathrm{Xa}$ binding site on human platelets. J. Biol. Chem. 253: 6908-6916.

2. Miletich, J. P., C. M. Jackson, and P. W. Majerus. 1977. Interaction of coagulation factor $\mathrm{Xa}$ with human platelets. Proc. Natl. Acad. Sci. USA. 74: 4033-4036.

3. Dahlback, B., and J. Stenflo. 1978. Binding of bovine coagulation factor $\mathrm{Xa}$ to platelets. Biochemistry. 17: 4938-4945.

4. Kane, W. H., M. L. J. Lindhout, C. M. Jackson, and P. W. Majerus. 1980. Factor Va-dependent binding of factor Xa to human platelets. J. Biol. Chem. 255: 11701174.

5. Tracy, P. B., J. M. Peterson, M. E. Nesheim, F. C. McDuffie, and K. G. Mann. 1979. Interaction of coagulation factor $\mathrm{V}$ and factor $\mathrm{Va}$ with platelets. J. Biol. Chem. 254: 10354-10361

6. Tracy, P. B., M. E. Nesheim, and K. G. Mann. 1981. Coordinate binding of factor $\mathrm{Va}$ and factor $\mathrm{Xa}$ to the unstimulated platelet. J. Biol. Chem. 256: 743-751.

7. Weiss, H. J., W. J. Vicic, B. A. Lages, and J. Rogers 1979. Isolated deficiency of platelet procoagulant activity. Am. J. Med. 67: 206-213.

8. Miletich, J. P., W. J. Kane, S. L. Hofmann, N. Stanford, and P. W. Majerus. 1979. Deficiency of factor Xa-factor
$\mathrm{Va}$ binding sites on the platelets of a patient with a bleeding disorder. Blood. 54: 1015-1022.

9. Esmon, C. T., W. G. Owen, and C. M. Jackson. 1974. A plausible mechanism for prothrombin activation by factor Xa, factor $\mathrm{Va}$, phospholipid, and calcium ions. $J$. Biol Chem. 249: 8045-8047.

10. Jackson, C. M., C. T. Esmon, S. N. Gitel, W. G. Owen, and R. A. Henricksen. 1975. The conversion of prothrombin to thrombin: the function of the propiece of prothrombin. In Prothrombin, and Related Coagulation Factors. H. C. Hemker and J. J. Veltkamp, editors. Leiden, Leiden, The Netherlands, 59-88.

11. Rosing, J., G. Tans, J. W. P. Govers-Riemslag, R. F. A. Zwaal, and H. C. Hemkin. 1979. The role of phospholipids and factor $\mathrm{Va}$ in the prothrombinase complex. $J$. Biol. Chem. 255: 274-283.

12. Marciniak, E. 1973. Factor $\mathrm{Xa}$ inactivation by antithrombin III. Evidence for biological stabilization of factor $\mathrm{Xa}$ by factor $\mathrm{V}$-phospholipid complex. Br. J. Haematol. 24: 391-400.

13. Walker, F. J., and C. T. Esmon. 1979. The effects of phospholipid and factor $\mathrm{Va}$ on the inhibition of factor $\mathrm{Xa}$ by antithrombin III. Biochem. Biophys. Res. Commun. 90: 641-647.

14. Lau, H. K., J. S. Rosenberg, D. L. Beeler, and R. D. Rosenberg. 1979. The isolation and characterization of a specific antibody population directed against the prothrombin activation fragments $F_{2}$ and $F_{1+2} . J$. Biol. Chem. 254: 8751-8761.

15. Jordan, R. E., L. V. Favreau, E. H. Braswell, and R. D. Rosenberg. 1982. Heparin with two binding sites for antithrombin or platelet factor 4. J. Biol. Chem. 257: 400-406.

16. Ryback, M. E., H. K. Lau, B. Tomkins, R. D. Rosenberg, and R. I. Handin. 1981. Relationship between platelet secretion and prothrombin cleavage in native whole blood. J. Clin. Invest. 68: 405-412.

17. Rodbard, D., R. H. Lenox, H. L. Wray, and D. Ramseth. 1976. Statistical characterization of the random errors in the radioimmunoassay dose-response variable. Clin. Chem. 22: 350-358.

18. Rodbard, D. 1974. Statistical quality control and routine data processing for radioimmunoassays and immunoradiometric assays. Clin. Chem. 20: 1255-1270.

19. Lewis, A. E. 1966. Biostatistics. Van Nostrand Reinhold Company, New York.

20. Harpel, P. C., and R. D. Rosenberg. 1976. $\alpha_{2}$-Macroglobulin and antithrombin-heparin cofactor: modulators of hemostatic and inflammatory reactions. Prog. $\mathrm{He}$ mostasis Thromb. 3: 145-189.

21. Teitel, J. M., K. A. Bauer, H. K. Lau, and R. D. Rosenberg. 1982. Studies of the prothrombin activation pathway utilizing radioimmunoassays for the $F_{2} / F_{1+2}$ fragment and thrombin-antithrombin complex. Blood. 59: 1086-1097.

22. Laurent, T. C. 1961. Studies on factionated heparin. Arch. Biochem. Biophys. 92: 224-231.

23. Lam, L. M., J. E. Silbert, and R. D. Rosenberg. 1976. The separation of active and inactive forms of heparin. Biochem. Biophys. Res. Commun. 69: 570-577.

24. Höök, M., I. Björk, J. Hopwood, and U. Lindahl. 1976. Anticoagulant activity of heparin: separation of high-activity and low-activity heparin species by affinity chromatography on immobilized antithrombin. FEBS (Fed. Eur. Biochem. Soc.). Lett. 66: 90-93.

25. Yin, E. T. 1974. Effect of heparin on the neutralization of factor $\mathrm{Xa}$ and thrombin by the plasma $\alpha_{2}$-globulin inhibitor. Thromb. Diath. Haemorrh. 33: 43-50. 